\title{
Giant gastroduodenal artery pseudoaneurysm as a pancreatic tumor and cause of acute bleeding into the digestive tract
}

\author{
Jacek Budzyński ${ }^{1}$, Grzegorz Meder², Karol Suppan \\ ${ }^{1}$ Department of Vascular and Internal Diseases, Faculty of Health Sciences, Nicolaus Copernicus University in Torun, \\ Ludwik Rydygier Collegium Medicum in Bydgoszcz, Poland \\ 2Department of Radiology and Diagnostic Imaging, Jan Biziel University Hospital No. 2, Bydgoszcz, Poland \\ ${ }^{3}$ Clinic of Vascular and Internal Diseases, Jan Biziel University Hospital No. 2, Bydgoszcz, Poland
}

Gastroenterology Rev 2016; 11 (4): 299-301

DOI: $10.5114 / p g .2016 .61478$

\begin{abstract}
Address for correspondence: Jacek Budzyński MD, PhD, Department of Vascular and Internal Diseases, Faculty of Health Sciences, Ludwik Rydygier Collegium Medicum, Nicolaus Copernicus University, 75 Ujejskiego St, 85-168 Bydgoszcz, Poland, phone: +48 5236551 48, +48 5236555 84, fax: +48 5236551 48, e-mail: budz@cps.pl
\end{abstract}

A 42-year-old woman was admitted to our department in October of 2014 due to abdominal pain that had begun 2 weeks earlier. On admission she did not present crucial abnormalities upon physical examination, other than a longitudinal scar in the anterior abdominal wall. Abdominal ultrasonography showed a peripancreatic tumor with signs of blood flow inside (Figure $1 \mathrm{~A}$ ). According to the patient's medical history, three hospitalizations had occurred on other wards due to: severe acute pancreatitis (August of 2012); a pancreatic pseudocyst that required drainage under endoscopic ultrasonography (EUS) control (October of 2013); and a tumor of the pancreatic head $(50 \times 53 \times$ $51 \mathrm{~mm}$ on abdominal computed tomography (CT)) and diabetes mellitus (September of 2014). During the last hospitalization the patient was referred to the Surgical Department because of the obscure character of the tumor of the pancreatic head and a family history of pancreatic cancer in order to perform the pancreato-duodenectomy. However, during the laparotomy the surgeons changed the primary plan and biopsies were taken only from the tumor. The results of the biopsies showed inflammatory and fibroid tissue in the pancreatic lesion (September 2014).

In our department, the initial suspicion of a vascular pancreatic lesion was confirmed using computed tomography angiography (angio-CT), which described: pseudoaneurysm of the gastroduodenal artery, $50 \times$ $40 \mathrm{~mm}$ in size (Figures $1 \mathrm{~B}-\mathrm{D}$ ), gastric fundus varices, liver steatosis with perfusion disturbances, and a dilated Wirsung duct. The female patient thus qualified for endovascular embolization. However, the procedure failed due to a problem with the catheterization of the vessel supplying the pseudoaneurysm. The patient was discharged without further complications. A second intervention was postponed due to radiation and the unchanged diameter of the pseudoaneurysm compared to September 2014. In November 2014, the patient was admitted due to signs of hemorrhage into the digestive tract with an obscure bleeding source, but without hemodynamic instability or significant decline in hemoglobin blood concentration. A second embolization of the gastroduodenal artery with coils placed distally and proximally to the canal supplying the lesion (to avoid retrograde filling) was performed with success. Control angiography (Figure $1 \mathrm{E}$ ) and ultrasonography (Figure $1 \mathrm{~F}$ ) performed 2 months after discharge did not detect any residual pseudoaneurysm.

Visceral artery aneurysms are divided into true or pseudoaneurysms [1]. Moreover, pseudoaneurysms are classified according to: the type of artery from which they originated, communication with the gastrointestinal tract, and potential exposure to pancreatic juice [1]. Visceral artery pseudoaneurysm appears in about $7-10 \%$ of patients with chronic pancreatitis and concern mainly splenic, gastroduodenal, and pancreaticoduodenal arteries [1]. The vascular lesions mentioned should be taken into consideration in the differential diagnosis of: (a) acute abdominal pain, especially in patients with shock and a history of chronic or acute pancreatitis, or abdominal surgery, (b) a tumor compressing the adjacent organs or vessels (e.g., portal or 

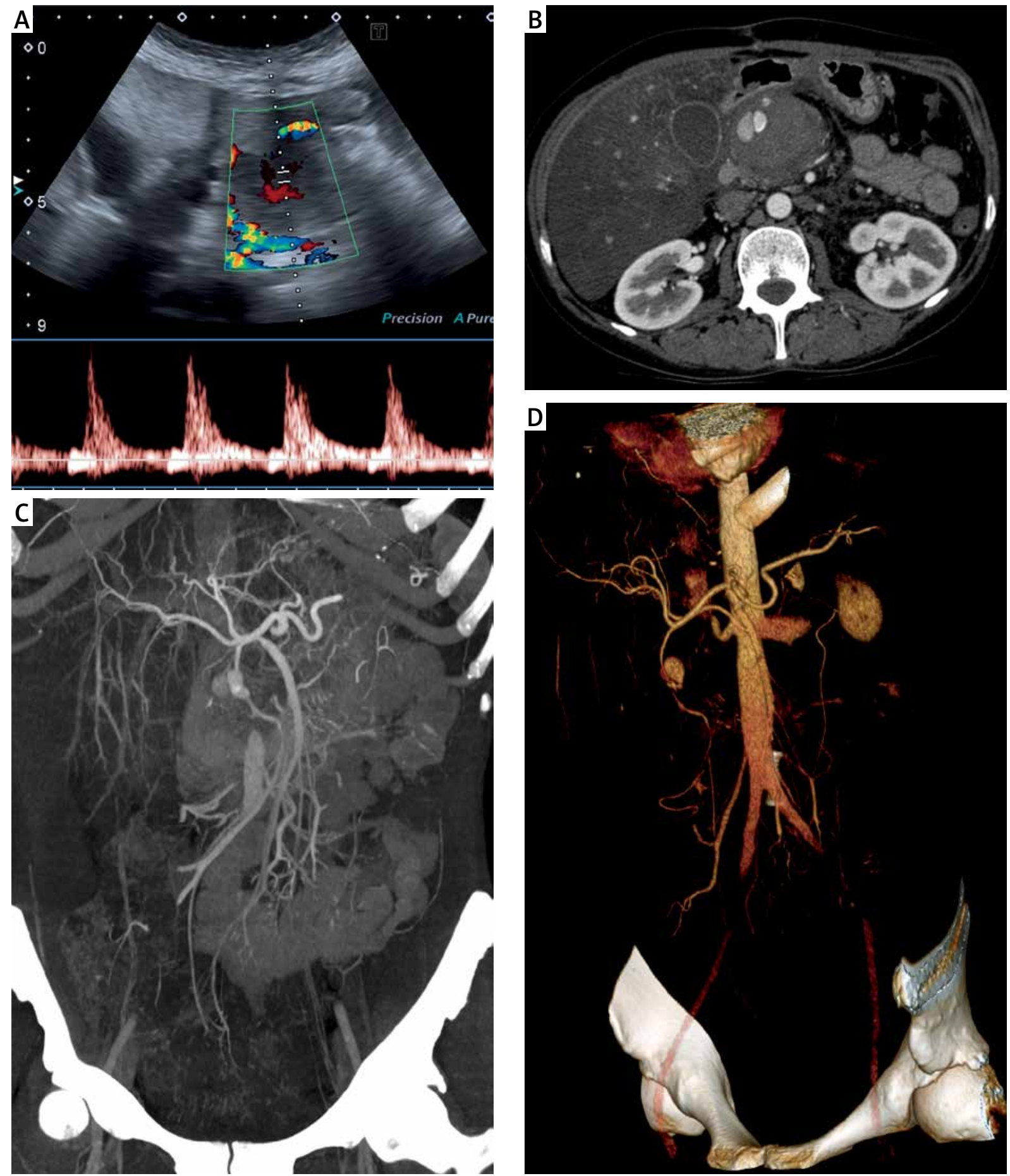

Figure 1. Giant aneurysm presentation: A - abdominal ultrasonography showed peripancreatic tumor with signs of blood flow inside; B - computed tomography angiography - pancreatic tumor with blood flow (pseudoaneurysm) in horizontal presentation; C - computed tomography angiography - pancreatic tumor with blood flow (pseudoaneurysm) in vertical presentation; D - computed tomography angiography $3 \mathrm{D}$ reconstruction of visceral vessels with pseudoaneurysm 

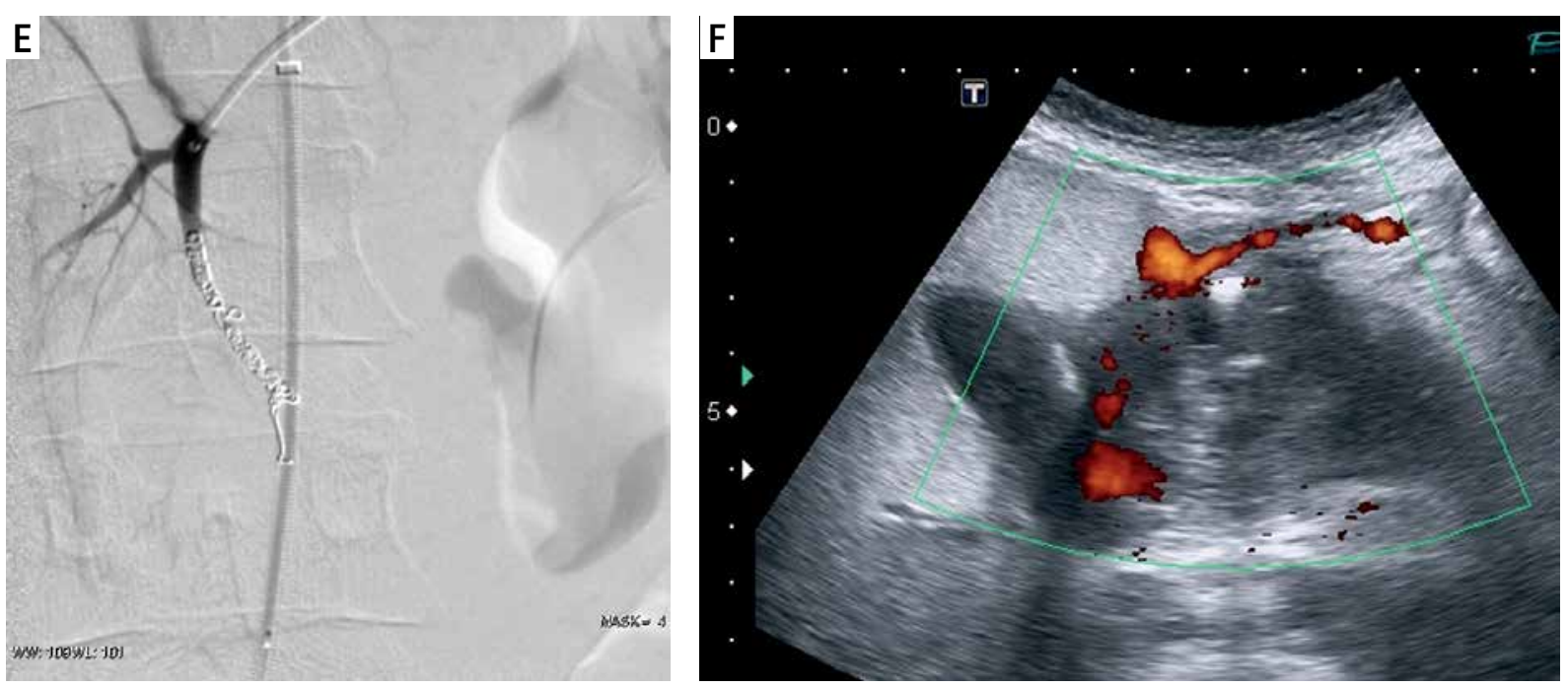

Figure 1. Cont. Giant aneurysm presentation: $\mathrm{E}$ - embolization of the gastroduodenal artery with coils placed distally and proximally to the canal supplying the lesion; $\mathbf{F}$ - abdominal ultrasonography 1 month after embolisation - pseudoaneurysmal sac without blood flow

splenic vein), as well as (c) bleeding into the peritoneal cavity, digestive tract, adjacent vein (arterio- venous fistula), pancreatic duct, biliary tract, or pseudocyst [1-3]. In each case, the visceral pseudoaneurysm should be treated, either by embolization (coils, covered stent, percutaneous or transcatheter thrombin injection) or by definitive surgery (Whipple procedure) [1-5]. After embolization the patients should be under observation due to $20-40 \%$ risk of early or late rebleeding [1]. For this reason and due to coexistence of not fully diagnosed tumor of the pancreatic head, the best treatment strategy would be pancreatoduodenectomy in this case [1]. However, the consulting surgeon suggested performing an intravascular embolization due to high (9\%) risk of complications and mortality (1.3\%) [3]. The patient remains under ambulatory control.

\section{Conflict of interest}

The authors declare no conflict of interest.

\section{References}

1. Pang TC, Maher R, Gananadha S, et al. Peripancreatic pseudoaneurysms: a management-based classification system. Surg Endosc 2014; 28: 2027-38.

2. Barbiero G, Battistel M, Susac A, Miotto D. Percutaneous thrombin embolization of a pancreatico-duodenal artery pseudoaneurysm after failing of the endovascular treatment. World J Radiol 2014; 6: 629-35.

3. Kukliński A, Batycki K, Matuszewski W, et al. Embolization of a large, symptomatic splenic artery pseudoaneurysm. Pol J Radiol 2014; 79: 194-8.
4. Barge JU, Lopera JE. Vascular complications of pancreatitis: role of interventional therapy. Korean J Radiol 2012; 13 Suppl 1: S45-55.

5. Czernik M, Stefańczyk L, Szubert W, et al. Endovascular treatment of pseudoaneurysms in pancreatitis. Videosurgery Miniinv 2014; 9: 138-44.

Received: 14.02 .2015

Accepted: 29.12 .2015 